\title{
PROTOSPIRURA HAMOSPICULATA N. SP. NÉMATODE PARASITE D'UN PANGOLIN AFRICAIN MANIS (PHOLIDOTUS) TEMMINCKI
}

\author{
Par M. NEVEU-LEMAIRE
}

La collection helminthologique du Laboratoire de Parasitologie de la Faculté de médecine de Paris contenait un flacon portant comme toute indication: "Parasites of Pholidotus temmincki " et renfermant 116 nématodes, dont 33 mâles et 83 femelles.

Ces nématodes appartiennent sans aucun doute à la famille des Spiruridæ Oerley, 1885 , à la sous-famille des Spirurinæ Railliet, 1915 et au genre Protospirura Seurat, 1914, dont nous donnons ici les caractères, en ajoutant quelques modifications à la diagnose primitive, afin de pouvoir y faire rentrer toutes les espèces connues :

" Corps régulièrement atténué antérieurement. Bouche présentant deux grandes lèvres latérales trilobées, chacun des lobes portant trois dents sur sa face interne. Vestibule buccal cylindrique et long. Esophage très long.

đ. Extrémité postérieure avec des ailes bien développées. Quatre paires de papilles subventrales préanales avec de courts pédoncules et une papille précloacale impaire. Deux paires de papilles postanales et un groupe de papilles plus petites près de l'extrémité caudale. Spicules égaux ou inégaux, le plus long pouvant être le droit ou le gauche. Gorgeret présent ou absent.

q. Queue courte. Vulve dans la région moyenne du corps. Utérus divergents. Ovipare. CEufs embryonnés au moment de la ponte. »

Après avoir examiné ces échantillons, je constatai qu'ils n'appartenaient à aucune des espèces de Protospirura décrites jusqu'ici et je propose de désigner cette nouvelle espèce sous le nom de $P$. hamospiculata (1), en raison de la terminaison en hameçon du spicule droit.

(1) De hamus, hameçon.

Annales de Parasitologie, T. V, $\mathrm{N}^{\circ} 2 .-1^{\text {er }}$ avril 1927 , p. 107-113. 

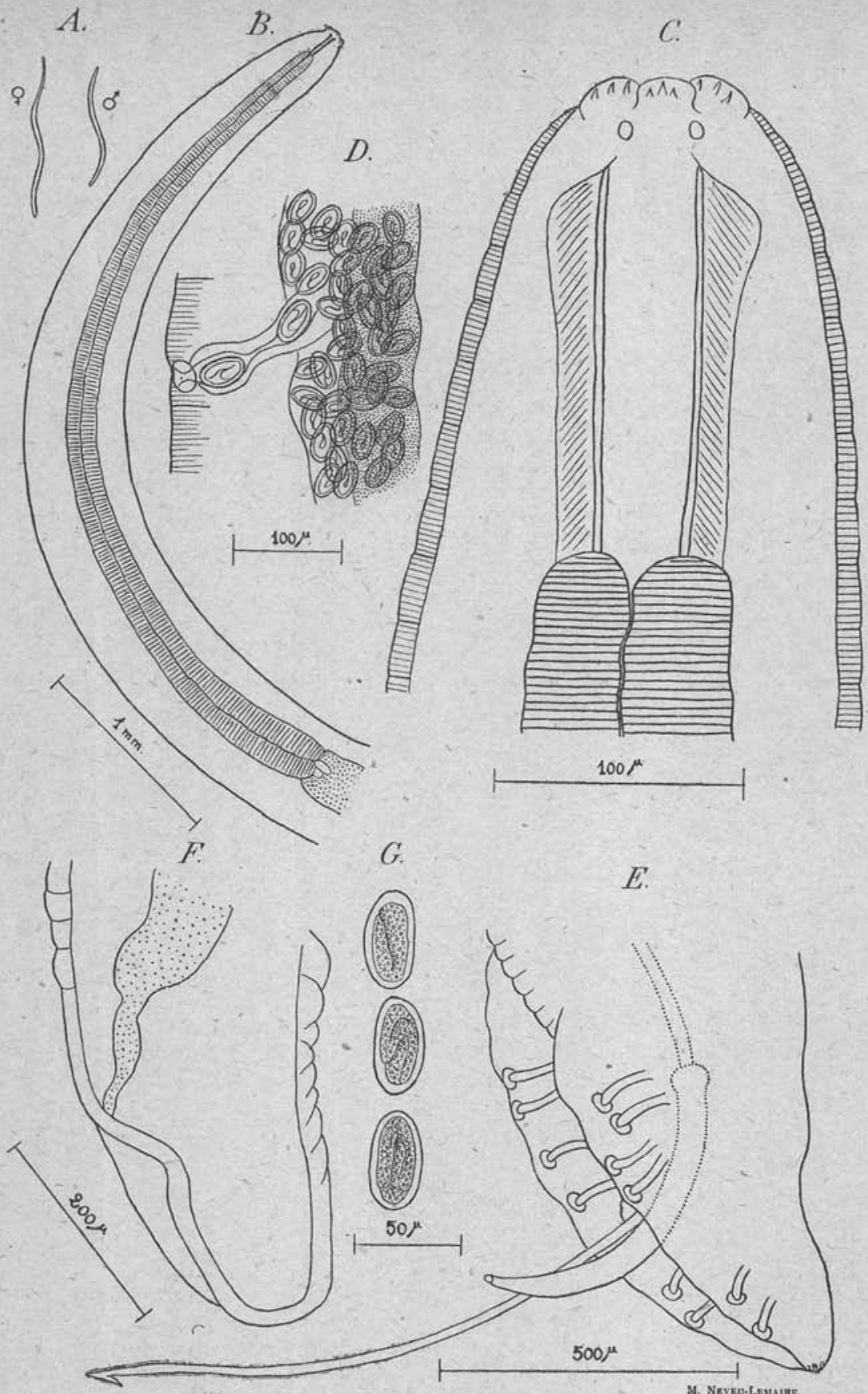

$E$.

Fig. - Protospirura hamospiculala n. sp. - $A$, mâle et femelle, grandeur naturelle; $B$, extrémité antérieure d'une femelle, montrant la grande longueur de l'cesophage; $C$, extrémité antérieure d'une femelle vue latéralement et très grossie; $D$, région vulvaire; $E$, extrémité postérieure d'un mâle vue du côté gauche ; $F$, extrémité postérieure d'une femelle vue du côté gauche; $G$, œufs. 


\section{Protospirura hamospiculata n. sp.}

Description. - Le corps est cylindroïde et trapu, comme chez les ascarides ; il est atténué antérieurement (fig. $A$ ) et de couleur blanc-jaunâtre. La cuticule est assez épaisse et présente des stries transversales très marquées, espacées les unes des autres et entre lesquelles on constate une striation beaucoup plus fine. La bouche est terminale et entourée de deux lèvres latérales formées chacune de trois lobes, un médian et deux latéraux; chacun de ces lobes porte sur sa face interne trois petites dents et les quatre lobes latéraux sont pourvus à leur base d'une papille. A la bouche fait suite un vestibule ou pharynx cylindrique, long d'environ $130 \mu$ (fig. $C$ ), qui débouche dans un osophage très long, pouvant atteindre chez la femelle adulte plus de 4 millimètres (fig. $B$ ). L'anneau nerveux est situé dans sa partie tout à fait antérieure, à environ $325 \mu$ de l'extrémité antérieure, chez le màle et à $350 \mathrm{ou} 400 \mu$ de cette extrémité chez la femelle. Le pore excréteur s'ouvre un peu en dessous, sur la face ventrale.

Mâle. - Le mâle a fréquemment son extrémité postérieure enroulée en spirale; il est un peu plus petit que la femelle et mesure de 15 à $17 \mathrm{~mm}$. de long sur un largeur maxima de 525 ¿ $570 \mu$. L'extrémité postérieure, conique, porte deux ailes bien développées sur lesquelles la cuticule forme des sortes de petites écailles. Le cloaque est situé à environ $300 \mu$ de l'extrémité. Il existe quatre paires -de papilles subventrales préanales pédonculées, une papille précloacale impaire, deux paires de papilles postanales pédonculées et quelques très petites papilles terminales. Les spicules sont inégaux; le spicule droit, beaucoup plus long et beaucoup plus ténu que le gauche, mesure $2 \mathrm{~mm}$., 3 et est terminé en hameçon; le spicule gauche, plus trapu et plus coürt, est disposé en arc, mesure $600 \mu$ et se termine en pointe mousse. Le gorgeret est absent (fig. $E$ ).

Femelle. - La femelle est ordinairement plus ou moins droite ; elle mesure de 18 à $23 \mathrm{~mm}$. de long sur une largeur maxima dé 590 à $680 \mu$. L'extrémité postérieure est conique ; l'anus est situé à 250 ou $300 \mu$ de cette extrémité (fig. $F$ ). La vulve se trouve dans le tiers antérieur du corps, à environ $9 \mathrm{~mm}$. de l'extrémité antérieure (fig. $D$ ). Les utérus sont divergents et remplis d'œufs en voie de segmentation ou déjà embryonnés. Les œufs, ovoïdes et légèrement asymétriques, mesurent de 44 à $46 \mu$ de long sur 23 à $24 \mu$ de large (fig. $G$ ). 
Hôte. - Ce nématode a été trouvé chez une espèce de pangolin habitant l'Afrique orientale et méridionale, le Manis (Pholidotus) temmincki Smuts, 1832. L'étiquette ne portait aucune indication de localisation du parasite, ni de localité.

Diagnose différentielle. - Nous indiquerons ici les caractères distinctifs des différentes espèces connues du genre Protospirura, puis nous donnerons, sous forme de tableau, leurs dimensions res. pectives.

1. Eufs très petits, mesurant au plus $16 \times 7 \mu$

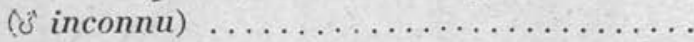

(Eufs beaucoup plus grands, mesurant de 47 à $60 \mu \times 30$ à $40 \mu \ldots \ldots \ldots \ldots \ldots \ldots \ldots$.

2. Appendice cuticulaire ventral dans la région de l'œsophage ( $q$ inconnue) $\ldots . . . \ldots \ldots$. Pas d'appendice cuticulaire ventral .......

3. Spicules égaux $\ldots \ldots \ldots \ldots \ldots \ldots \ldots \ldots \ldots$

Spicules inégaux $\ldots \ldots \ldots \ldots \ldots \ldots \ldots \ldots$

4. Vuive située en arrière du milieu du corps . Vulve située en avant du milieu du corps.

5. Spicules mesurant respectivement 480 et

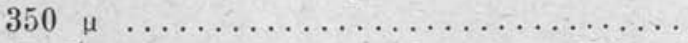

Spicules mesurant respectivement 830 et 420

6. Diflérence de longueur entre les deux spiculess, inférieure à $300 \mu \ldots \ldots \ldots \ldots \ldots$.

Différence de longueur entre les deux spicules, supérieure à $500 \mu \ldots \ldots \ldots \ldots$.

7. Grande espèce, mesurant jusqu'à $43 \mathrm{~mm}$. chez le $\delta$ et $100 \mathrm{~mm}$. chez la $q \ldots \ldots \ldots$. Petite espèce, mesurant au plus $30 \mathrm{~mm}$. chez le $\delta$ et $40 \mathrm{~mm}$. chez la $q \ldots \ldots \ldots$.

8. Spicule droit terminé en hameçon ........ Les deux spicules terminés en pointe plus ou moins mousse ..................

9. Grande espèce, mesurant jusqu'à $45 \mathrm{~mm}$. chez le ot et $85 \mathrm{~mm}$. chez la $q \ldots \ldots \ldots \ldots$ Petite espèce, dont le $\delta$ mesure $23 \mathrm{~mm} .2$ (q inconnue)

P. labiodentata.

P. guianensis.

P. bonnei.

P. muricola.

P. numidica.

P. columbiana.

P. muris.

P. hamospiculata.

P. ascaroidea.

P. gracilis. 


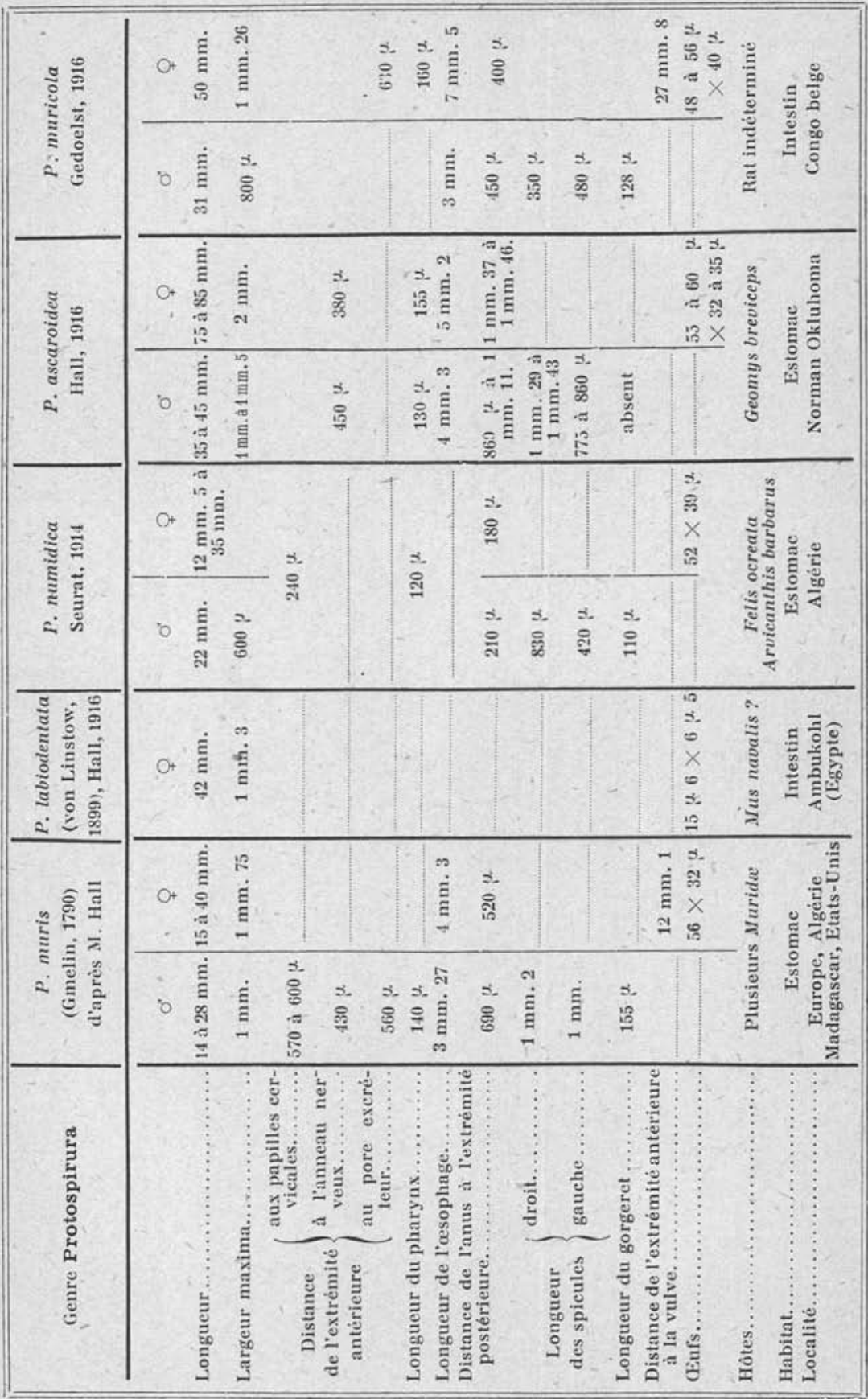




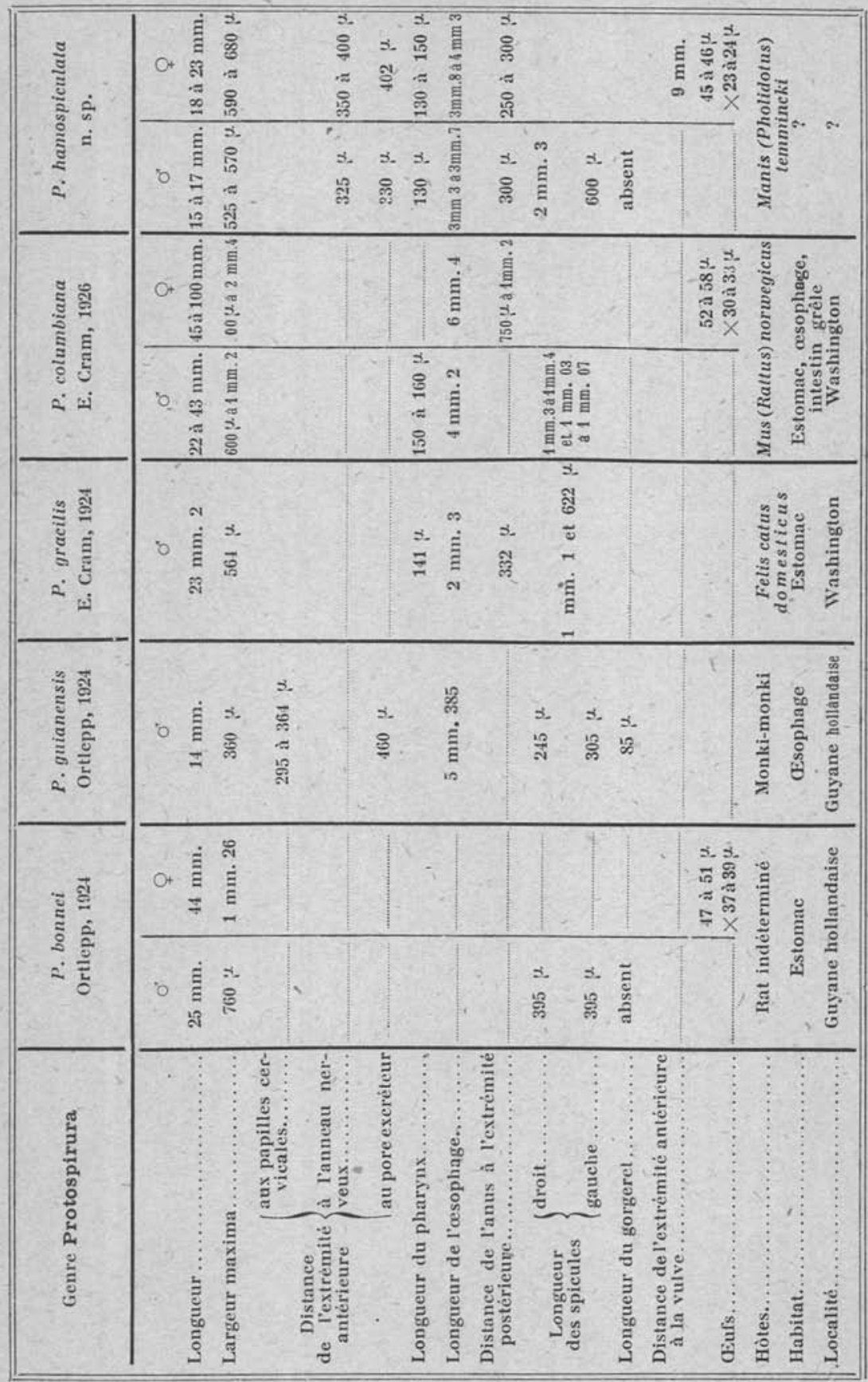




\section{BIBLIOGRAPHIE}

Baylis (H-A.) et Daubney (R.). - A synopsis of the families and genera of Nematoda. London, 1926.

Craм (Eloïse). - A new nematode, Prolospirura gracilis, from the cat. Journ. amer. vet. med. assoc., LXV, 1924.

- A new nematode from the rat and its life history. Proc. of the U.S. national Museum, Washington, LXVIII, 1926, p. 1.

Gedoelst (L.). - Notes sur la faune parasitaire du Congo belge. Rev. zool. africaine, V, 1916, p. 1-90.

Gmelin (J.-F.). - Systema natura, etc., part 6, Vermss. Lipsiæ, 1790.

HaL. (M.-C.). - Nématode parasites of mammals of the orders Rodentia, Lagomorpha and Hyracoidea. Proc. of the U. S. national Museum, Washington, L, 1916, p. 1-258.

Linstow (O, von), - Nematoden aus der Berliner zoologischen Sammlung. Mitt. a. d. zool. Mns.,'Berlin, I, 1899, p. 3 .

OrtLepp (R.-.J). - Helminths from Dutch Guiana. Journ, of helminthology, II, 1924 , p. 15.

Rudolphi (C.-A). - Entozoorum synopsis cui accedeut mantissa duplex et indices locupletissimi. Berolini, 1819.

Seurat (G.-L.). - Sur un nouveau spiroptère du chat ganté. C. $R$. soc. biol., LXXVII, 1914, p. 344-347.

- Sur l'habitat normal et les affinités du Protospirura numidica Seurat. $C$. $R$. soc. biol., LXXIX, I916, p. 143-146.

Yorke (W.) et MAPLESTONE (P.-A.). - The nematode parasites of vertebrates. London, 1926.

Laboratoire de Parasilologie de la Faculté de médecine de Paris. 